Bangladesh J. Plant Taxon. 26(2): 285-298, 2019 (December)

(C) 2019 Bangladesh Association of Plant Taxonomists

\title{
ANGIOSPERMS IN GOBINDAGANJ UPAZILA OF GAIBANDHA DISTRICT, BANGLADESH
}

\author{
PriyanKa SARKER AND A.H.M. MAhbubur Rahman ${ }^{1}$ \\ Plant Taxonomy Laboratory, Department of Botany, Faculty of Life and Earth Sciences, \\ University of Rajshahi, Rajshahi 6205, Bangladesh
}

Keywords: Diversity, Angiosperm taxa, Gobindaganj Upazila, Gaibandha District, Bangladesh

\begin{abstract}
Angiosperms at Gobindaganj Upazila of Gaibandha district, Bangladesh was studied from January to December 2018. An extensive floristic survey and angiosperms collection have been made throughout the study area. A total of 295 species belonging to 246 genera under 89 families were recorded. Plant habit analysis shows that herbs, shrubs, climbers and trees are represented by $47.45 \%, 15.93 \%, 12.20 \%$ and $24.40 \%$, respectively. Distribution of angiosperm species in the families shows variation. Asteraceae is the most dominant family represented by 25 species, followed by Fabaceae (19 species), Euphorbiaceae (18 species), Cucurbitaceae (17 species), Acanthaceae (11 species), Solanaceae (11 species), Amaranthaceae (10 species) and Apocynaceae (10 species). 44 families are represented by a single species each while 37 families are represented by 2 to 8 species each. Status of occurrence has been recorded for proper conservation management and sustainable utilization of the taxa which show 218 $(73.89 \%)$ to be common, $63(21.35 \%)$ as rare, $10(3.38 \%)$ as vulnerable, and $4(1.35 \%)$ are found as endangered in the study area. For each species scientific name, voucher number, Bangla name, English name, habit, status of occurrence and flowering time were recorded.
\end{abstract}

\section{Introduction}

The flowering plants, also known as angiosperms, Angiospermae or Magnoliophyta, are the most diverse group of land plants, with 64 orders, 416 families, approximately 13,000 known genera and 300,000 known species (Christenhusz and Byng, 2016). Angiosperms are seedproducing plants like the gymnosperms and can be distinguished from the gymnosperms by a series of synapomorphies (derived characteristics). These characteristics include flowers, endosperm within the seeds, and the production of fruits that contain the seeds. Etymologically, angiosperm means a plant that produces seeds within an enclosure; they are fruiting plants, although more commonly referred to as flowering plants. The ancestors of flowering plants diverged from gymnosperms around 245-202 million years ago, and the first flowering plants known to exist are from 160 million years ago. They diversified enormously during the Lower Cretaceous and became widespread around 120 million years ago, but replaced conifers as the dominant trees only around 60-100 million years ago (Lindley, 1830).

The angiosperms provide valuable pharmaceuticals. With the exception of antibiotics, almost all medicines are either derived directly from compounds produced by angiosperms or, if synthesized, were originally discovered in angiosperms. This includes some vitamins (e.g., vitamin C, originally extracted from fruits); aspirin, originally from the bark of willows (Salix; Salicaceae); narcotics (e.g., opium and its derivatives from the opium poppy, Papaver

\footnotetext{
${ }^{1}$ Corresponding author: E-mail: drrahmanahmm@ru.ac.bd
} 
somniferum; Papaveraceae); and quinine from Cinchona (Rubiaceae) bark. Sonic angiosperm compounds that are highly toxic to humans have proved to be effective in the treatment of certain forms of cancer, such as acute leukemia (vincristine from the Madagascar periwincle, Catharanthus roseus, Apocynaceae), and of heart problems (digitalis from foxglove, Digitalis purpurea, Plantaginaceae). Muscle relaxants derived from curare (Strychnos toxifera, Loganiaceae) are used during open-heart surgery (Naik, 2003).

Over the last few decades several attempts have been made on the floristic studies in Bangladesh, particularly in the forest and protected areas (Khan and Afza, 1968; Khan and Banu, 1972; Khan and Hassan, 1984; Rahman and Hassan, 1995; Uddin et al., 2013; Khan and Huq, 2001; Uddin and Hassan, 2010; Tutul et al., 2010; Arefin et al., 2011; Uddin and Hassan, 2012). Studies on angiosperm flora in different districts and Upazilas of Bangladesh are limited (Islam et al., 2009; Rahman et al., 2013; Moniruzzaman et al., 2012; Rahman and Alam, 2013). However, there has been no floristic study in Gobindaganj Upazila of Gaibandha district, Bangladesh.

\section{Materials and Methods}

Study area: Gobindaganj is an upazila of Gaibandha district under the division of Rangpur. It is one of the largest upazila in Bangladesh including 17 unions and 1 municipality. Gobindaganj is located at $25.1333^{\circ} \mathrm{N} 3^{\prime}$ and $89.391^{\circ} \mathrm{E}$. It is bounded by Ghoraghat and Polashbari upazilas on the north, Sonatala and Shibgonj upazilas on the south, Saghatta and Polashbari uazilas on the east, Panchbibi and Kalia upazilas on the west. One fourth of the total area of the upazila is included in the Barind Tract. It has 79464 households and a total area of $481.66 \mathrm{Sq}$. Km. As of 2011 Bangladesh census, Gobindaganj has a population of 714591 . Males constitute $50.89 \%$ of the population, and females $49.11 \%$. This upazila's adult population is 205204 . Soil texture was determined by hydrometer method and soil $\mathrm{P}^{\mathrm{H}}$ was measured in a 1:2.5. Soil water suspension measured by glass clatrode $\mathrm{pH}$ meter. This is the best soil for the growth of various plants. The study area has tropical monsoon climate. It is characterized by hot humid summers and generally mild winters and rainfall. The summer season commerce early in the March with the cessation of the Northerly wind. The winter season (November-January) which is cool and with little rainfall; the summer season (June-October) is warm and with no rainfall. The maximum monthly temperature can reach up to $37.78^{\circ} \mathrm{C}$ during April and minimum monthly temperature $7.78^{\circ} \mathrm{C}$ during January (BPC, 2001).

Methodology: The work is based on fresh materials collected during twenty seven visits to Gobindhaganj Upazila of Gaibandha, Bangladesh from January 2018 to December 2018 to cover the seasonal variations. The visits covered all types of habitats, particular river bank, slope, village grove, fruit gardens and roadsides of the study area. Each trip lasted for eight days. Plant parts with either flower or fruits were collected using traditional herbarium techniques to make voucher specimens for documentation. Field identification of the collected specimens was confirmed comparing with herbarium specimens Rajshahi University Herbarium. Standard literature such as Hooker (1877), Prain (1903), and Ahmed et al. (2008-2009) were consulted for identification. For nomenclature Pasha and Uddin (2013) and Huq (1986) were also consulted. The specimens are deposited in the Herbarium, Department of Botany, Rajshahi University, Bangladesh for future reference.

\section{Results and Discussion}

Angiosperm diversity at Gobindaganj upazila of Gaibandha district, Bangladesh was investigated during January to December 2018. A total of 295 species belonging to 246 genera 
under 89 families were recorded (Table 1). Of these, Magnoliopsida (Dicotyledons) is represented by 261 species under 213 genera and 73 families while Liliopsida (Monocotyledons) is represented by 34 species under 33 genera and 16 families. Habit analysis shows that herbs, shrubs, climbers and trees are represented by $47.45 \%, 15.93 \%, 12.20 \%, 24.40 \%$ species, respectively (Fig.1).

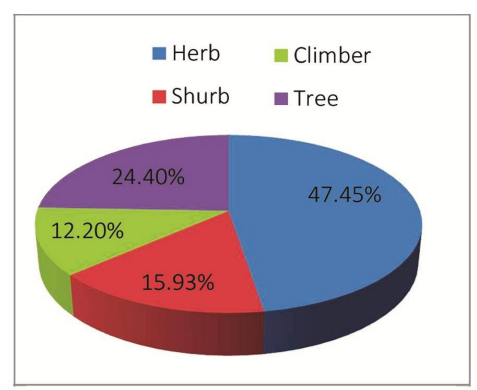

Fig. 1. Recorded Magnoliopsida plants habit diversity in the study area.

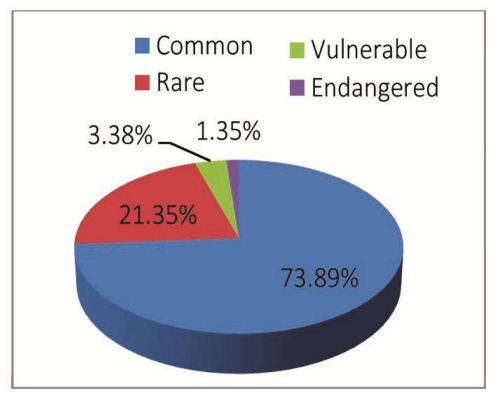

Fig. 2. Recorded Magnoliopsida plants status of occurrence in the study area.

Distribution of angiosperm species in the families shows variation. Asteraceae is the dominant family represented by 25 species, followed by Fabaceae (19 species), Euphorbiaceae (18 species), Cucurbitaceae (17 species), Acanthaceae (11 species), Solanaceae (11 species), Amaranthaceae (10 species) and Apocynaceae (10 species) (Table 1; Fig. 3). 44 families are represented by a single species each, while 37 families are represented by 2-8 species each. Status of occurrence has been recorded for proper conservation management and sustainable utilization of the taxa, which show $218(73.89 \%)$ to be common, $63(21.35 \%)$ as rare, $10(3.38 \%)$ as vulnerable and 4 $(1.35 \%)$ as endangered in the study area (Fig. 2).

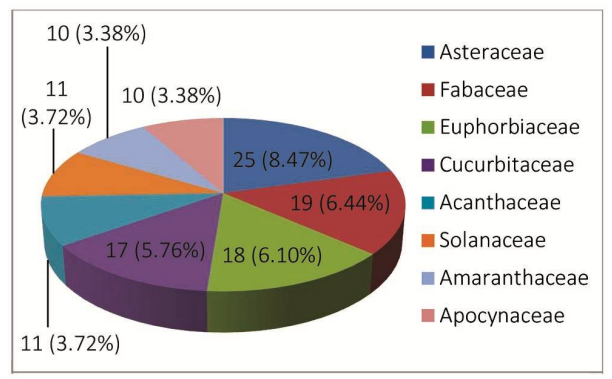

Fig. 3. Dominant plant families in the study area.

Based on the study, a preliminary list of angiosperm diversity at Gobindaganj upazila of Gaibandha district, Bangladesh is recorded. A total of 295 species belonging to 246 Genera under 89 families were found (Table 1). The collected information is comparable with the result of other studies in Bangladesh. A total of 243 species belonging to 195 genera under 95 families were recorded in Khagrachhari district (Islam et.al, 2009). A total of 374 species belonging to 264 genera under 84 families were recorded in Lawachara National Park (Uddin and Hassan, 2010). A total of 153 species belonging to 120 genera under 52 families were recorded in Runctia sal Forest (Tutul et. al, 2010). A total of 245 species belonging to 183 genera and 72 families were documented in Hobiganj district (Anefin et al., 2011). A total of 425 species belonging to 321 
Table 1. Angiosperm Taxa in Gobindagaj Upazila of Gaibandha District, Bangladesh

\begin{tabular}{|c|c|c|c|c|c|}
\hline $\begin{array}{l}\text { Scientific name and Voucher } \\
\text { number }\end{array}$ & Bangla name & Family & Habit & $\begin{array}{c}\text { Status of } \\
\text { occurrence }\end{array}$ & $\begin{array}{l}\text { Flowering } \\
\text { time }\end{array}$ \\
\hline \multicolumn{6}{|l|}{ MAGNOLIOPSIDA } \\
\hline Michelia champaca L., PS 19 & Champa & Magnoliaceae & Tree & $\mathrm{R}$ & Mar-Apr \\
\hline Annona reticulata L., PS 29 & Nona, Ata & Annonaceae & Tree & $\mathrm{C}$ & Oct-Jan \\
\hline A. squamosa L., PS 37 & Sarifa & Annonaceae & Tree & $\mathrm{C}$ & Mar-Jul \\
\hline $\begin{array}{l}\text { Polyalthia longifolia (Sonn.) Thw., } \\
\text { PS } 21\end{array}$ & Debdaru & Annonaceae & Tree & $\mathrm{C}$ & Mar-Oct \\
\hline $\begin{array}{l}\text { Cinnamomum tamala Nees \& } \\
\text { Eberm, PS } 49\end{array}$ & Tejpata & Lauraceae & Tree & $\mathrm{R}$ & Feb-Oct \\
\hline C. verum J. S. Presl, PS 131 & Daruchini & Lauraceae & Tree & $\mathrm{R}$ & Jan-Mar \\
\hline $\begin{array}{l}\text { Litsea glutinosa (Lour.) Rob., PS } \\
200\end{array}$ & Menda & Lauraceae & Tree & V & Apr-Jan \\
\hline $\begin{array}{l}\text { Peperomia pellucida (L.) H.B. \& } \\
\text { K., PS } 44\end{array}$ & Luchi Pata & Piperaceae & Herb & $\mathrm{C}$ & Jul-Sep \\
\hline $\begin{array}{l}\text { Nymphaea nouchali Burm f., PS } \\
132\end{array}$ & Nilsapla & Nymphaeaceae & Herb & $\mathrm{C}$ & Jun-Oct \\
\hline $\begin{array}{l}\text { Stephania japonica (Thunb.) Miers., } \\
\text { PS } 201\end{array}$ & Akanadi & Menispermaceae & Climber & $\mathrm{R}$ & Jan-Dec \\
\hline $\begin{array}{l}\text { Tinospora cordifolia (Willd.) } \\
\text { Hook.f. \& Thoms., PS } 45\end{array}$ & Gulancha & Menispermaceae & Climber & $\mathrm{R}$ & Jan-Oct \\
\hline Argemone mexicana L., PS 133 & Sheyalkata & Papaveraceae & Herb & $\mathrm{C}$ & Feb-Jun \\
\hline $\begin{array}{l}\text { Trema orientalis (L.) Blume, PS } \\
202\end{array}$ & Jibon & Ulmaceae & Tree & $\mathrm{C}$ & Jan-Jun \\
\hline $\begin{array}{l}\text { Artocarpus heterophyllus Lamk., } \\
\text { PS } 266\end{array}$ & Kathal & Moraceae & Tree & $\mathrm{C}$ & Feb-Jul \\
\hline A. lacucha Buch-Ham, PS 309 & Dewa & Moraceae & Tree & $\mathrm{V}$ & Apr-Jun \\
\hline Ficus benghalensis L., PS 332 & Bot & Moraceae & Tree & $\mathrm{C}$ & May-Aug \\
\hline F. hispida L.f., PS 346 & Khoksa & Moraceae & Tree & $\mathrm{C}$ & Apr-Sep \\
\hline F. racemosa L., PS 59 & Jagdumur & Moraceae & Tree & $\mathrm{C}$ & Sep-Nov \\
\hline F. religiosa L., PS 134 & Pakur & Moraceae & Tree & $\mathrm{C}$ & Mar-Oct \\
\hline Sterblus asper Lour., PS 203 & Sheora & Moraceae & Tree & $\mathrm{R}$ & Feb-Jun \\
\hline Cannabis sativa L., PS 267 & Ganja & Cannabaceae & Herb & $\mathrm{R}$ & Jan-Dec \\
\hline $\begin{array}{l}\text { Pouzolzia zeylanica (L.) Benn., PS } \\
310\end{array}$ & Kullaruki & Urticaceae & Herb & $\mathrm{C}$ & Jan-Dec \\
\hline $\begin{array}{l}\text { Bougainvillea spectabilis Willd., PS } \\
333\end{array}$ & Baganbilash & Nyctaginaceae & Climber & $\mathrm{R}$ & Nov-Feb \\
\hline Mirabilis jalapa L., PS 58 & $\begin{array}{l}\text { Sondha } \\
\text { maloti }\end{array}$ & Nyctaginaceae & Herb & $\mathrm{C}$ & Jan-Dec \\
\hline Chenopodium album L., PS 135 & Bothua & Chenopodiaceae & Herb & $\mathrm{C}$ & Dec-Mar \\
\hline C. ambrosioides L., PS 204 & Banbotua & Chenopodiaceae & Herb & $\mathrm{C}$ & Jan-Dec \\
\hline Spinacia oleracea L., PS 268 & Palongshak & Chenopodiaceae & Herb & $\mathrm{C}$ & Feb-Mar \\
\hline Achyranthes aspera L., PS 311 & Aparg & Amaranthaceae & Herb & $\mathrm{C}$ & Jan-Dec \\
\hline $\begin{array}{l}\text { Aerva lanata (L.) Juss. ex Schult, } \\
\text { PS } 334\end{array}$ & Chaya & Amaranthaceae & Herb & $\mathrm{C}$ & Apr-Jul \\
\hline Alternanthera sessilis R.Br., PS 399 & Chanshi & Amaranthaceae & Herb & $\mathrm{C}$ & Jan-Dec \\
\hline $\begin{array}{l}\text { A. philoxeroides (Mart.) Griseb., PS } \\
61\end{array}$ & Malancha & Amaranthaceae & Herb & $\mathrm{C}$ & Mar-Jun \\
\hline
\end{tabular}


Table 1 Contd.

\begin{tabular}{|c|c|c|c|c|c|}
\hline $\begin{array}{l}\text { Scientific name and Voucher } \\
\text { number }\end{array}$ & Bangla name & Family & Habit & $\begin{array}{l}\text { Status of } \\
\text { occurrence }\end{array}$ & $\begin{array}{l}\text { Flowering } \\
\text { time }\end{array}$ \\
\hline Amaranthus dubius Mart., PS 136 & Daata & Amaranthaceae & Herb & $\mathrm{C}$ & Feb-Oct \\
\hline A. spinosus L., PS 205 & Katanotey & Amaranthaceae & Herb & $\mathrm{C}$ & Jan-Dec \\
\hline A. viridis L., PS 269 & Notyshak & Amaranthaceae & Herb & $\mathrm{C}$ & Jan-Dec \\
\hline A. tricolor L., PS 312 & Lalshak & Amaranthaceae & Herb & $\mathrm{C}$ & Jan-Dec \\
\hline Celosia cristata L., PS 335 & Morogful & Amaranthaceae & Herb & $\mathrm{C}$ & Jan-Dec \\
\hline Dirgeria arvensis Forsk, PS 347 & Gungatika & Amaranthaceae & Herb & $\mathrm{V}$ & Feb-Jun \\
\hline Portulaca oleracea L., PS 348 & Baranunia & Portulacaceae & Herb & $\mathrm{C}$ & May-Jul \\
\hline P. quadrifida L., PS 67 & Chotonumia & Portulacaceae & Herb & $\mathrm{C}$ & May-Dec \\
\hline Basella rubra L., PS 137 & Pushak & Basellaceae & Climber & $\mathrm{C}$ & Nov-Feb \\
\hline Mollugo pentaphylla L., PS 206 & Khetpapra & Molluginaceae & Herb & $\mathrm{C}$ & Jun-Jan \\
\hline $\begin{array}{l}\text { Polycarpon prostratum (Forsk) } \\
\text { Asch. \& Sch., PS } 270\end{array}$ & Ghima & Caryophyllaceae & Herb & $\mathrm{C}$ & Dec-Feb \\
\hline $\begin{array}{l}\text { Persicaria hydropiper (L.) Spach., } \\
\text { PS } 313\end{array}$ & Biskatali & Polygonaceae & Herb & $\mathrm{C}$ & Aug-Apr \\
\hline Dillenia indica L., PS 63 & Chalta & Dilleniaceae & Tree & $\mathrm{R}$ & May-Oct \\
\hline $\begin{array}{l}\text { Elaeocarpus tectorius (Lour.) Poir., } \\
\text { PS } 138\end{array}$ & Jolpai & Elaeocarpaceae & Tree & $\mathrm{R}$ & May-Oct \\
\hline Corchorus capsularis L., PS 271 & Deshipat & Tiliaceae & Shrub & $\mathrm{C}$ & Aug-Feb \\
\hline Grewia tiliifolia Vahl., PS 51 & Pholsa & Tiliaceae & Tree & $\mathrm{R}$ & Sep-May \\
\hline Abroma augusta (L.) L.f., PS 139 & Ulatkambal & Sterculiaceae & Shrub & $\mathrm{V}$ & Jun-Dec \\
\hline Bombax ceiba L., PS 207 & Shimul & Bombacaceae & Tree & $\mathrm{C}$ & Feb-Apr \\
\hline $\begin{array}{l}\text { Abelmoschus esculentus (L.) } \\
\text { Moench, PS } 272\end{array}$ & Bhindi & Malvaceae & Herb & $\mathrm{C}$ & Jan-Dec \\
\hline $\begin{array}{l}\text { Abutilon indicum (L.) Sweet., PS } \\
314\end{array}$ & Petari & Malvaceae & Herb & $\mathrm{C}$ & Jul-Apr \\
\hline Gossypium hirsutum L., PS 336 & Karpas Tula & Malvaceae & Shrub & $\mathrm{C}$ & Oct-Jan \\
\hline Hibiscus rosa-sinensis L., PS 349 & Joba & Malvaceae & Shrub & $\mathrm{C}$ & Jan-Dec \\
\hline Sida cordifolia L., PS 69 & Berela & Malvaceae & Herb & $\mathrm{C}$ & Sep-Dec \\
\hline $\begin{array}{l}\text { Barringtonia acutangula (L.) } \\
\text { Gaerth, PS } 140\end{array}$ & Hijal & Lecythidaceae & Tree & $\mathrm{E}$ & May-Sep \\
\hline Carica papaya L., PS 208 & Pape & Caricaceae & Tree & $\mathrm{C}$ & Jan-Dec \\
\hline $\begin{array}{l}\text { Benincasa hispida (Thurb.) Cogn., } \\
\text { PS } 273\end{array}$ & Chalkumra & Cucurbitaceae & Climber & $\mathrm{C}$ & May-Nov \\
\hline $\begin{array}{l}\text { Citrullus lanatus (Thunb.) Mat. \& } \\
\text { Nak., PS } 70\end{array}$ & Tormuj & Cucurbitaceae & Climber & $\mathrm{C}$ & Mar-Sep \\
\hline $\begin{array}{l}\text { Coccinia grandis (L.) Voigt., PS } \\
141\end{array}$ & Telakucha & Cucurbitaceae & Climber & $\mathrm{C}$ & Mar-Dec \\
\hline Cucumis melo L., PS 209 & Bangi & Cucurbitaceae & Climber & $\mathrm{C}$ & Mar-Jul \\
\hline C. sativus L., PS 274 & Sosha & Cucurbitaceae & Climber & $\mathrm{C}$ & Apr-Sep \\
\hline Cucurbita maxima Duch, PS 315 & Mistikumra & Cucurbitaceae & Climber & $\mathrm{C}$ & Mar-Aug \\
\hline $\begin{array}{l}\text { Gymnopetalum cochinchinense } \\
\text { (Lour.) Kurz., PS } 337\end{array}$ & Bati Jhinga & Cucurbitaceae & Climber & $\mathrm{R}$ & Jul-Dec \\
\hline $\begin{array}{l}\text { Lagenaria siceraria (Monila) } \\
\text { Standl., PS } 71\end{array}$ & Panilau & Cucurbitaceae & Climber & $\mathrm{C}$ & Feb-May \\
\hline Luffa acutangula (L.) Roxb., PS 142 & Jhinga & Cucurbitaceae & Climber & $\mathrm{C}$ & Apr-Oct \\
\hline
\end{tabular}


Table 1 Contd.

\begin{tabular}{|c|c|c|c|c|c|}
\hline $\begin{array}{l}\text { Scientific name and Voucher } \\
\text { number }\end{array}$ & Bangla name & Family & Habit & $\begin{array}{l}\text { Status of } \\
\text { occurrence }\end{array}$ & $\begin{array}{l}\text { Flowering } \\
\text { time }\end{array}$ \\
\hline Luffa cylindrica (L.) Roem, PS 210 & Dhundul & Cucurbitaceae & Climber & $\mathrm{C}$ & Jun-Dec \\
\hline Momordica charantia L., PS 275 & Korolla & Cucurbitaceae & Climber & $\mathrm{C}$ & May-Oct \\
\hline $\begin{array}{l}\text { M. cochinchinensis (Lour.) Spreng., } \\
\text { PS } 72\end{array}$ & Kakrol & Cucurbitaceae & Climber & $\mathrm{C}$ & Jul-Nov \\
\hline $\begin{array}{l}\text { Mukia maderaspatana (L.) M. } \\
\text { Roem., PS } 143\end{array}$ & Agmuki & Cucurbitaceae & Climber & $\mathrm{R}$ & Jun-Dec \\
\hline Trichosanthes anguina L., PS 211 & Chichinga & Cucurbitaceae & Climber & $\mathrm{C}$ & Apr-Jun \\
\hline T. cucumerina L., PS 276 & Banchichinga & Cucurbitaceae & Climber & $\mathrm{C}$ & Apr-Jun \\
\hline T. dioica Roxb., PS 73 & Potol & Cucurbitaceae & Climber & $\mathrm{C}$ & Apr-Sep \\
\hline T. tricuspidata Lour., PS 144 & Makal & Cucurbitaceae & Climber & $\mathrm{R}$ & Jul-Dec \\
\hline Cleome viscosa L., PS 212 & Hurhuria & Capparaceae & Herb & $\mathrm{R}$ & Jan-Dec \\
\hline Brassica napus L., PS 277 & Sarisha & Brassicaceae & Herb & $\mathrm{C}$ & Mar- Jul \\
\hline $\begin{array}{l}\text { B. oleracea L.var. capitata L., PS } \\
316\end{array}$ & Badhakopi & Brassicaceae & Herb & $\mathrm{C}$ & Nov-Apr \\
\hline $\begin{array}{l}\text { B. oleracea L.var. botrytis L., PS } \\
338\end{array}$ & Phulkopi & Brassicaceae & Herb & $\mathrm{C}$ & Nov-Apr \\
\hline Raphanus sativus L., PS 74 & Mula & Brassicaceae & Herb & $\mathrm{C}$ & Jan-May \\
\hline Moringa oleifera Lamk., PS 145 & Sajna & Moringaceae & Tree & $\mathrm{C}$ & Jan-Dec \\
\hline $\begin{array}{l}\text { Manilkara zapota (L.) P. van } \\
\text { Royen, PS } 213\end{array}$ & Sofeda & Sapotaceae & Tree & $\mathrm{C}$ & May-Jun \\
\hline Mimusops elengi L., PS 75 & Bokul & Sapotaceae & Tree & $\mathrm{C}$ & Mar-Jun \\
\hline Diospyros blancoi A. DC., PS 214 & Bilatigab & Ebenaceae & Tree & $\mathrm{R}$ & May-Jul \\
\hline $\begin{array}{l}\text { D. malabarica (Desr.) Kostel., PS } \\
278\end{array}$ & Deshi Gab & Ebenaceae & Tree & $\mathrm{C}$ & May-Jul \\
\hline D. montana Roxb., PS 317 & Tamal & Ebenaceae & Tree & V & Mar-May \\
\hline $\begin{array}{l}\text { Bryophyllum pinnatum (Lamk.) } \\
\text { Oken, PS } 76\end{array}$ & Pathorkuchi & Crassulaceae & Herb & $\mathrm{C}$ & Nov-Jan \\
\hline $\begin{array}{l}\text { Kalanchoe laciniata (L.) Pers., PS } \\
146\end{array}$ & Himsagor & Crassulaceae & Herb & $\mathrm{R}$ & Jan-Mar \\
\hline Rosa centifolia L., PS 215 & Golap & Rosaceae & Shrub & $\mathrm{C}$ & Jan-Dec \\
\hline $\begin{array}{l}\text { Acacia auriculiformis A. Cunn. ex. } \\
\text { Benth \& Hook, PS } 279\end{array}$ & Akasmoni & Mimosaceae & Tree & $\mathrm{C}$ & Jun-Feb \\
\hline A. catcechu (L.f) Wild., PS 318 & Khair & Mimosaceae & Tree & $\mathrm{R}$ & Mar-Dec \\
\hline A. nilotica (L.) Del., PS 339 & Babla & Mimosaceae & Tree & $\mathrm{C}$ & Apr-Aug \\
\hline $\begin{array}{l}\text { Albizia procera (Roxb.) Benth, PS } \\
350\end{array}$ & Silkoroi & Mimosaceae & Tree & $\mathrm{C}$ & Jan-Dec \\
\hline Mimosa pudica L., PS 77 & Lojjaboti & Mimosaceae & Herb & $\mathrm{C}$ & Sep-Dec \\
\hline Bauhinia acuminata L., PS 147 & $\begin{array}{l}\text { Sada } \\
\text { Kanchan }\end{array}$ & Caesalpiniaceae & Tree & $\mathrm{R}$ & Jan - Dec \\
\hline Cassia fistula L., PS 216 & Badorlathi & Caesalpiniaceae & Tree & $\mathrm{C}$ & Mar-Apr \\
\hline Delonix regia (Boyer) Raf, PS 280 & Krishnochura & Caesalpiniaceae & Tree & $\mathrm{C}$ & Apr-Sep \\
\hline Senna alata (L.) Roxb., PS 78 & Dadmardan & Caesalpiniaceae & Shrub & $\mathrm{R}$ & Sep-Jan \\
\hline S. sophera (L.) Roxb., PS 148 & Kalkashunda & Caesalpiniaceae & Shrub & $\mathrm{C}$ & Dec-Mar \\
\hline Tamarindus indica L., PS 217 & Tentul & Caesalpiniaceae & Tree & $\mathrm{C}$ & Apr-Dec \\
\hline Abrus precatorius L., PS 79 & Kuch & Fabaceae & Climber & $\mathrm{R}$ & Jul-Sep \\
\hline
\end{tabular}


Table 1 Contd.

\begin{tabular}{|c|c|c|c|c|c|}
\hline $\begin{array}{l}\text { Scientific name and Voucher } \\
\text { number }\end{array}$ & Bangla name & Family & Habit & $\begin{array}{c}\text { Status of } \\
\text { occurrence }\end{array}$ & $\begin{array}{l}\text { Flowering } \\
\text { time }\end{array}$ \\
\hline Arachis hypogea L., PS 149 & Chinabadam & Fabaceae & Herb & $\mathrm{C}$ & Mar-Dec \\
\hline Cajanus cajan (L.) Millsp, PS 218 & Arhar & Fabaceae & Shrub & $\mathrm{C}$ & Dec-Apr \\
\hline Clitoria ternatea L., PS 281 & Aparajita & Fabaceae & Climber & $\mathrm{C}$ & Jan-Dec \\
\hline Crotalaria pallida Ait., PS 80 & Jhun-Jhuni & Fabaceae & Herb & $\mathrm{C}$ & May-Dec \\
\hline Dalbergia sissoo Roxb., PS 150 & Sissoo Gachh & Fabaceae & Tree & $\mathrm{C}$ & Mar-Jun \\
\hline $\begin{array}{l}\text { Desmodium gangeticum (L.) DC., } \\
\text { PS } 219\end{array}$ & Salpani & Fabaceae & Herb & $\mathrm{C}$ & Apr-Nov \\
\hline D. triflorum (L.) DC., PS 282 & Kudalia & Fabaceae & Herb & $\mathrm{C}$ & Jan-Dec \\
\hline Erythrina variegata L., PS 81 & Madar & Fabaceae & Tree & $\mathrm{C}$ & Feb-May \\
\hline $\begin{array}{l}\text { Lablab purpureus (L.) Sweet, PS } \\
151\end{array}$ & Shim & Fabaceae & Climber & $\mathrm{C}$ & Nov-Mar \\
\hline Lathyrus sativus L., PS 220 & Khesari & Fabaceae & Herb & $\mathrm{C}$ & Feb-Sep \\
\hline Lens culinaris Medic., PS 82 & Masur & Fabaceae & Herb & $\mathrm{C}$ & Dec-Mar \\
\hline Melilotus alba Desr., PS 152 & Sada Methi & Fabaceae & Herb & $\mathrm{R}$ & Mar-Oct \\
\hline $\begin{array}{l}\text { Pongamia pinnata (L.) Pierre, PS } \\
221\end{array}$ & Karanja & Fabaceae & Tree & $\mathrm{R}$ & Mar-Jul \\
\hline $\begin{array}{l}\text { Sesbania bispinosa (Jacq.) Wight., } \\
\text { PS } 283\end{array}$ & Dhaincha & Fabaceae & Shrub & $\mathrm{C}$ & May-Oct \\
\hline Uraria picta (Jacq.) Desv., PS 83 & Shankar Jata & Fabaceae & Herb & $\mathrm{R}$ & Jun-Dec \\
\hline Visia sativa L., PS 153 & Ankari & Fabaceae & Herb & $\mathrm{C}$ & Jul-Nov \\
\hline Vigna mungo (L.) Hepper, PS 222 & Mashkalai & Fabaceae & Herb & $\mathrm{C}$ & Nov-Jan \\
\hline V. unguiculata (L.) Walp., PS 284 & Borboti & Fabaceae & Climber & $\mathrm{C}$ & Jan-Dec \\
\hline $\begin{array}{l}\text { Lagerstroemia speciosa (L.) Pers., } \\
\text { PS } 319\end{array}$ & Jarul & Lythraceae & Tree & $\mathrm{C}$ & Apr-Aug \\
\hline Lawsoria inermis L., PS 84 & Mehedi & Lythraceae & Shrub & $\mathrm{C}$ & Jun-Dec \\
\hline Trapa bispinosa Roxb., PS 154 & Paniphal & Trapaceae & Herb & $\mathrm{C}$ & Jun-Sep \\
\hline Eucalyptus citriodora Hook, PS 223 & Eucalyptus & Myrtaceae & Tree & $\mathrm{C}$ & Jan-Dec \\
\hline Psidium guajava L., PS 85 & Peyara & Myrtaceae & Tree & $\mathrm{C}$ & Jan-Dec \\
\hline $\begin{array}{l}\text { Syzygium cumini (L.) Skeels., PS } \\
155\end{array}$ & Jam & Myrtaceae & Tree & $\mathrm{C}$ & Mar-Jun \\
\hline S. jambos (L.) Alston, PS 224 & Golapjam & Myrtaceae & Tree & $\mathrm{R}$ & Mar-Jun \\
\hline $\begin{array}{l}\text { S. samarangense (Blume) Merr \& } \\
\text { Perry, PS } 86\end{array}$ & Jamrul & Myrtaceae & Tree & $\mathrm{C}$ & Feb-Mar \\
\hline Punica granatum L., PS 156 & Dalim & Punicaceae & Shrub & $\mathrm{C}$ & Jan-Dec \\
\hline $\begin{array}{l}\text { Ludwigia adscendens (L.) Hara, PS } \\
225\end{array}$ & Kesordam & Onagraceae & Herb & $\mathrm{C}$ & Mar-Dec \\
\hline Quisqualis indica L, PS 320 & Madhabi Lata & Combretaceae & Shrub & $\mathrm{C}$ & Jan-May \\
\hline $\begin{array}{l}\text { Terminalia arjuna (Roxb. ex. DC) } \\
\text { Wight \& Arn., PS } 87\end{array}$ & Arjun & Combretaceae & Tree & $\mathrm{C}$ & Apr-Jul \\
\hline T. chebula Retz., PS 157 & Haritaki & Combretaceae & Tree & $\mathrm{R}$ & May-Jun \\
\hline Santalum album L., PS 226 & $\begin{array}{l}\text { Shwet } \\
\text { Chandan }\end{array}$ & Santalaceae & Tree & $\mathrm{E}$ & Feb-Jul \\
\hline $\begin{array}{l}\text { Dendrophthe falcata (L.f.) Etting., } \\
\text { PS } 285\end{array}$ & Bandha & Loranthaceae & $\begin{array}{l}\text { Parasitic } \\
\text { Shrub }\end{array}$ & $\mathrm{R}$ & Jan-Dec \\
\hline Acalypha indica L., PS 88 & Muktajhuri & Euphorbiaceae & Herb & $\mathrm{C}$ & Dec-Apr \\
\hline
\end{tabular}


Table 1 Contd.

\begin{tabular}{|c|c|c|c|c|c|}
\hline $\begin{array}{l}\text { Scientific name and Voucher } \\
\text { number }\end{array}$ & Bangla name & Family & Habit & $\begin{array}{c}\text { Status of } \\
\text { occurrence }\end{array}$ & $\begin{array}{l}\text { Flowering } \\
\text { time }\end{array}$ \\
\hline Baccaurea ramiflora Lour., PS 158 & Latkan & Euphorbiaceae & Tree & $\mathrm{R}$ & Jun-Sep \\
\hline $\begin{array}{l}\text { Codiaeum variegatum (L.) A. Juss., } \\
\text { PS } 227\end{array}$ & Patabahar & Euphorbiaceae & Shrub & $\mathrm{C}$ & Jan-Dec \\
\hline $\begin{array}{l}\text { Croton bonplandianus Baill, PS } \\
286\end{array}$ & Banmorich & Euphorbiaceae & Herb & $\mathrm{C}$ & Jan-Dec \\
\hline Euphorbia hirta L. , PS 321 & Dudhiya & Euphorbiaceae & Herb & $\mathrm{C}$ & Jan-Dec \\
\hline E. nerifolia $\mathrm{L} .$, PS 340 & Mansasij & Euphorbiaceae & Shrub & $\mathrm{R}$ & Jun-Nov \\
\hline $\begin{array}{l}\text { E. pulcherrima Willd. ex Klotz., PS } \\
89\end{array}$ & Lal Pata & Euphorbiaceae & Shrub & $\mathrm{R}$ & Dec-Mar \\
\hline E. thymifolia L., PS 159 & Swetkan & Euphorbiaceae & Herb & $\mathrm{R}$ & Jan-Dec \\
\hline Jatropha curcas L., PS 228 & Jamalgota & Euphorbiaceae & Shrub & $\mathrm{R}$ & Sep-Dec \\
\hline J. gossypifolia L., PS 287 & Lalbherenda & Euphorbiaceae & Shrub & $\mathrm{C}$ & Apr-Aug \\
\hline Manihot esculenta Crantz., PS 90 & Kasava & Euphorbiaceae & Shrub & $\mathrm{C}$ & Sep-Jan \\
\hline Phyllanthus emblica L., PS 160 & Amloki & Euphorbiaceae & Tree & $\mathrm{R}$ & Mar-Sep \\
\hline P. niruri L., PS 229 & Bhuiamla & Euphorbiaceae & Herb & $\mathrm{C}$ & Aug-Oct \\
\hline P. reticulatus Poir, PS 288 & Chitki & Euphorbiaceae & Shrub & $\mathrm{C}$ & Mar-Oct \\
\hline P. urinaria $\mathrm{L}$., PS 91 & Hazarmari & Euphorbiaceae & Herb & $\mathrm{C}$ & Apr-Oct \\
\hline Ricinus communis L., PS 161 & Bherenda & Euphorbiaceae & Shrub & $\mathrm{C}$ & Jan-Dec \\
\hline Tragia involucrata L., PS 230 & Bichuti & Euphorbiaceae & Herb & $\mathrm{E}$ & Oct-Jan \\
\hline Trewia nodiflora L., PS 289 & Batul, Latim & Euphorbiaceae & Tree & $\mathrm{R}$ & Feb-Aug \\
\hline Zizyphus mauritiana Lamk., PS 322 & Boroi & Rhamnaceae & Tree & $\mathrm{C}$ & Sep-Jan \\
\hline Vitis trifolia (L.) Domin., PS 341 & Amallata & Vitaceae & Climber & $\mathrm{R}$ & Jan-Dec \\
\hline Litchi chinensis Sonn., PS 92 & Lichu & Sapindaceae & Tree & $\mathrm{C}$ & Apr-Jun \\
\hline Magnifera indica L., PS 162 & Aam & Anacardiaceae & Tree & $\mathrm{C}$ & Jan-Apr \\
\hline Spondias pinnata (L.f) kurz, PS 231 & Aamra & Anacardiaceae & Tree & $\mathrm{C}$ & Feb-Jun \\
\hline Azadirachta indica A. Juss., PS 290 & Neem & Meliaceae & Tree & $\mathrm{C}$ & Mar-Jul \\
\hline Swietenia mahagoni Jacq., PS 93 & Mahagoni & Meliaceae & Tree & $\mathrm{C}$ & Apr-Nov \\
\hline Aegle marmelos (L.) Corr., PS 163 & Bel & Rutaceae & Tree & $\mathrm{C}$ & Apr-Dec \\
\hline $\begin{array}{l}\text { Citrus aurantifolia (Christm. \& } \\
\text { Panzer) Swingle., PS } 232\end{array}$ & Labu & Rutaceae & Shrub & $\mathrm{C}$ & Mar-Sep \\
\hline $\begin{array}{l}\text { Citrus maxima (Burm.) Merr., PS } \\
291\end{array}$ & Jambura & Rutaceae & Tree & $\mathrm{C}$ & Feb-Nov \\
\hline $\begin{array}{l}\text { Glycosmis pentaphylla (Retz.) } \\
\text { A.DC., PS } 323\end{array}$ & Datmajani & Rutaceae & Shrub & $\mathrm{R}$ & Jan-Dec \\
\hline Limonia acidissima L., PS 94 & Kothbel & Rutaceae & Tree & $\mathrm{C}$ & Feb-Dec \\
\hline $\begin{array}{l}\text { Murraya paniculata (L.) Jack, PS } \\
164\end{array}$ & Kamini & Rutaceae & Shrub & $\mathrm{C}$ & Mar-Jan \\
\hline Averrhoa carambola L., PS 233 & Kamranga & Oxalidaceae & Tree & $\mathrm{C}$ & Sep-Mar \\
\hline Oxalis corniculata L., PS 292 & Amrul & Oxalidaceae & Herb & $\mathrm{C}$ & Sep-May \\
\hline $\begin{array}{l}\text { Impatiens balsamina Thusmb, PS } \\
324\end{array}$ & Dupati & Balsaminaceae & Herb & $\mathrm{C}$ & Mar-Oct \\
\hline $\begin{array}{l}\text { Centella asiatica (L.) Urban, PS } \\
342\end{array}$ & Thankuni & Apiaceae & Herb & $\mathrm{C}$ & Jan-Dec \\
\hline Coriandrum sativum L., PS 95 & Dhoney & Apiaceae & Herb & $\mathrm{C}$ & Dec-Feb \\
\hline Daucus carota L., PS 165 & Gajor & Apiaceae & Herb & $\mathrm{C}$ & May-Aug \\
\hline
\end{tabular}


Table 1 Contd.

\begin{tabular}{|c|c|c|c|c|c|}
\hline $\begin{array}{l}\text { Scientific name and Voucher } \\
\text { number }\end{array}$ & Bangla name & Family & Habit & $\begin{array}{l}\text { Status of } \\
\text { occurrence }\end{array}$ & $\begin{array}{l}\text { Flowering } \\
\text { time }\end{array}$ \\
\hline Exacum pedunculatum L., PS 234 & Chirattam & Gentianaceae & Herb & $\mathrm{R}$ & Feb-Apr \\
\hline Allamanda cathartica L., PS 293 & Alkananda & Apocynaceae & Shrub & $\mathrm{C}$ & Jan-Dec \\
\hline Alstonia scholaris (L.) R. Br., PS 96 & Chatim & Apocynaceae & Tree & $\mathrm{R}$ & Nov-May \\
\hline Carissa carandas L., PS 166 & Karamcha & Apocynaceae & Herb & $\mathrm{C}$ & Jan-Dec \\
\hline $\begin{array}{l}\text { Catharanthus roseus (L.) G. Don., } \\
\text { PS } 235\end{array}$ & Nayantara & Apocynaceae & Herb & $\mathrm{C}$ & Jan-Dec \\
\hline $\begin{array}{l}\text { Holarrhena antidysenterica (L.) } \\
\text { Wall. ex Decne, PS } 97\end{array}$ & Kurchi & Apocynaceae & Tree & $\mathrm{V}$ & Apr-Jan \\
\hline Nerium oleander L., PS 167 & Korobi & Apocynaceae & Shrub & $\mathrm{C}$ & Jan-May \\
\hline Plumeria alba L., PS 236 & Katgolap & Apocynaceae & Tree & $\mathrm{R}$ & May-Nov \\
\hline $\begin{array}{l}\text { Rauvolfia serpentina (L.) Benth ex } \\
\text { Kurz., PS } 294\end{array}$ & Sarpagandha & Apocynaceae & Herb & $\mathrm{R}$ & Jan-Dec \\
\hline $\begin{array}{l}\text { Tabernaemontana divaricata (L.) } \\
\text { R.Br.ex.Roem \& Schult, PS } 325\end{array}$ & Tagor & Apocynaceae & Shrub & $\mathrm{R}$ & May-Jan \\
\hline Thevetia peruviana Pers., PS 98 & Haldekarabi & Apocynaceae & Tree & $\mathrm{C}$ & Jan-Dec \\
\hline $\begin{array}{l}\text { Calotropis gigantea (L.) R.Br., PS } \\
168\end{array}$ & Akondo & Asclepiadaceae & Shrub & $\mathrm{C}$ & Apr-May \\
\hline C. procera (Ait.) R. Br., PS 237 & Akondo & Asclepiadaceae & Shrub & $\mathrm{C}$ & Apr-May \\
\hline Capsicum frutescens L., PS 99 & Morich & Solanaceae & Herb & $\mathrm{C}$ & Jan-Dec \\
\hline Cestrum nocturnum L., PS 169 & Hasnahena & Solanaceae & Shrub & $\mathrm{C}$ & Jan-Dec \\
\hline Datura metel L., PS 238 & Dhatura & Solanaceae & Shrub & $\mathrm{C}$ & Jan-Dec \\
\hline $\begin{array}{l}\text { Lycopersicon esculentum Mill., PS } \\
295\end{array}$ & Tomato & Solanaceae & Herb & $\mathrm{C}$ & Sep-Apr \\
\hline $\begin{array}{l}\text { Nicotiana plumbaginifolia } \text { Viv., PS } \\
326\end{array}$ & Bontamak & Solanaceae & Herb & $\mathrm{C}$ & Jan-Dec \\
\hline Physalis minima L., PS 343 & Kopalftka & Solanaceae & Herb & $\mathrm{C}$ & Jan-Dec \\
\hline Solanum melongena L., PS 100 & Begun & Solanaceae & Herb & $\mathrm{C}$ & Oct-Feb \\
\hline S. nigrum L., PS 170 & Titbegun & Solanaceae & Herb & $\mathrm{C}$ & Jan-Dec \\
\hline S. tuberosum L., PS 239 & Golalu & Solanaceae & Herb & $\mathrm{C}$ & Oct-Feb \\
\hline S. torvum Swartz., PS 296 & Gota Begun & Solanaceae & Shrub & $\mathrm{C}$ & Dec-Feb \\
\hline S. virginianum L., PS 101 & Kantakari & Solanaceae & Herb & $\mathrm{C}$ & Oct-Feb \\
\hline $\begin{array}{l}\text { Evolvulus nummularius (L.) L., PS } \\
171\end{array}$ & Bhuiokra & Convolvulaceae & Herb & $\mathrm{C}$ & Jan-Dec \\
\hline Ipomoea aquatica Forssk, PS 240 & Kalmishak & Convolvulaceae & Climber & $\mathrm{C}$ & Jan-Oct \\
\hline I. batatas (L.) Lamk., PS 297 & Mistialu & Convolvulaceae & Climber & $\mathrm{C}$ & Jan-Dec \\
\hline $\begin{array}{l}\text { I. fistulosa Mart. ex. Choisy in DC., } \\
\text { PS } 102\end{array}$ & Dholkalmi & Convolvulaceae & Shrub & $\mathrm{C}$ & Jan-Dec \\
\hline Cuscuta reflexa Roxb., PS 172 & Swarnolata & Cuscutaceae & Climber & $\mathrm{C}$ & Aug-Dec \\
\hline $\begin{array}{l}\text { Nymphoides indicum (L.) O, } \\
\text { Kuntze, PS } 241\end{array}$ & Panchuli & Menyanthaceae & Herb & $\mathrm{C}$ & Oct-Feb \\
\hline Cordia dichotoma Forst., PS 103 & Boula & Boraginaceae & Tree & $\mathrm{V}$ & Feb-Aug \\
\hline Heliotropium indicum L., PS 173 & Hatishur & Boraginaceae & Herb & $\mathrm{C}$ & Jan-Dec \\
\hline $\begin{array}{l}\text { Clerodendrum viscosum Vent., PS } \\
242\end{array}$ & Bhat & Verbenaceae & Shrub & $\mathrm{C}$ & Jan-Jul \\
\hline C. inerme (L.) Grentn, PS 298 & Bamunhati & Verbenaceae & Shrub & $\mathrm{C}$ & Jul-Nov \\
\hline
\end{tabular}


Table 1 Contd.

\begin{tabular}{|c|c|c|c|c|c|}
\hline $\begin{array}{l}\text { Scientific name and Voucher } \\
\text { number }\end{array}$ & Bangla name & Family & Habit & $\begin{array}{c}\text { Status of } \\
\text { occurrence }\end{array}$ & $\begin{array}{l}\text { Flowering } \\
\text { time }\end{array}$ \\
\hline Duranta repens L., PS 104 & Katamehedi & Verbenaceae & Shrub & $\mathrm{C}$ & Jan-Dec \\
\hline Lantana camara L., PS 174 & Chotra & Verbenaceae & Shrub & $\mathrm{C}$ & Jan-Dec \\
\hline $\begin{array}{l}\text { Lippia alba (Mill.) Briton } e t \\
\text { Wilson., PS } 243\end{array}$ & Boraokra & Verbenaceae & Shrub & $\mathrm{C}$ & Jan-Dec \\
\hline Phyla nodiflora (L.) Greene, PS 299 & Khudiokra & Verbenaceae & Herb & $\mathrm{C}$ & Jan-Dec \\
\hline Tectona grandis L.f., PS 105 & Shegun & Verbenaceae & Tree & $\mathrm{C}$ & Jul-Nov \\
\hline Vitex negundo L., PS 175 & Nisinda & Verbenaceae & Shrub & $\mathrm{R}$ & Apr-Feb \\
\hline $\begin{array}{l}\text { Anisomeles indica (L.) Kuntz., PS } \\
244\end{array}$ & Gobura & Lamiaceae & Herb & $\mathrm{R}$ & Oct-Jul \\
\hline Hyptis suaveolens (L.) Poit., PS 327 & Tokma & Lamiaceae & Herb & $\mathrm{V}$ & Jan-Dec \\
\hline Leonurus sibiricus L., PS 106 & Roktodron & Lamiaceae & Herb & $\mathrm{C}$ & Jan-Dec \\
\hline Leucas aspera (Willd) Link, PS 176 & Shetodron & Lamiaceae & Herb & $\mathrm{C}$ & Jan-Dec \\
\hline Mentha viridis L., PS 245 & Pudina & Lamiaceae & Herb & $\mathrm{C}$ & Jul-Sep \\
\hline Ocimum tenuiflorum L., PS 300 & Tulsi & Lamiaceae & Herb & $\mathrm{R}$ & Jan-Dec \\
\hline O. americanum L., PS 107 & Bon Tulsi & Lamiaceae & Herb & $\mathrm{C}$ & Jun-Feb \\
\hline Jasminum sambac (L.) Ait., PS 177 & Beli & Oleaceae & Shrub & $\mathrm{C}$ & Mar-Jul \\
\hline Nyctanthes arbor-tristis L., PS 246 & Sheuli & Oleaceae & Shrub & $\mathrm{C}$ & Aug-Sep \\
\hline $\begin{array}{l}\text { Bacopa monnieri (L.) Pannel, PS } \\
301\end{array}$ & Brammishak & Scrophulariaceae & Herb & $\mathrm{V}$ & May-Dec \\
\hline Scoparia dulcis L., PS 328 & Bondone & Scrophulariaceae & Herb & $\mathrm{C}$ & Jan-Dec \\
\hline $\begin{array}{l}\text { Andrographis paniculata (Burm.f.) } \\
\text { Wall ex Ness., PS } 108\end{array}$ & Kalamegh & Acanthaceae & Herb & $\mathrm{C}$ & Jan-Mar \\
\hline Barleria prionitis L., PS 178 & Kanta-janti & Acanthaceae & Herb & $\mathrm{C}$ & Nov-Feb \\
\hline $\begin{array}{l}\text { Eranthemum pulchellum Andre, PS } \\
247\end{array}$ & Shukh Murali & Acanthaceae & Shrub & $\mathrm{R}$ & Feb-Apr \\
\hline $\begin{array}{l}\text { Hemigraphis hirta (Vahl) T. } \\
\text { Anders., PS } 302\end{array}$ & Buriana & Acanthaceae & Herb & $\mathrm{C}$ & Jan-Jul \\
\hline $\begin{array}{l}\text { Hygrophila schulli (Buch.-Ham.) } \\
\text { M.R. \& S.N.Almeida, PS } 109\end{array}$ & Kulekharha & Acanthaceae & Herb & $\mathrm{R}$ & Oct-Jan \\
\hline Justicia adhatoda L., PS 179 & Basok & Acanthaceae & Shrub & $\mathrm{R}$ & Jan-Apr \\
\hline J. gendarussa Burm. f., PS 248 & $\begin{array}{l}\text { Jagath } \\
\text { madan }\end{array}$ & Acanthaceae & Herb & $\mathrm{C}$ & Apr-Aug \\
\hline $\begin{array}{l}\text { Nelsonia canescens (Lamk.) } \\
\text { Spreng., PS } 110\end{array}$ & Paramul & Acanthaceae & Herb & $\mathrm{C}$ & Oct-Feb \\
\hline Ruellia tuberosa L., PS 180 & Chatpoty & Acanthaceae & Herb & $\mathrm{C}$ & Jan-Dec \\
\hline $\begin{array}{l}\text { Rungia pectinata (L.) Ness in DC., } \\
\text { PS } 249\end{array}$ & Pindi & Acanthaceae & Herb & $\mathrm{C}$ & Nov-May \\
\hline $\begin{array}{l}\text { Thunbergia grandiflora (Roxb. ex } \\
\text { Rottler) Roxb., PS } 303\end{array}$ & Nillata & Acanthaceae & Climber & $\mathrm{R}$ & Jan-Dec \\
\hline Sesamum indicum L., PS 329 & Til & Pedaliaceae & Herb & $\mathrm{C}$ & Feb-Oct \\
\hline Tabebuia aurea F.T, PS 344 & Tobebia & Bignoniaceae & Tree & $\mathrm{E}$ & Jan-Dec \\
\hline $\begin{array}{l}\text { Gardenia augusta (L.) Merr., PS } \\
345\end{array}$ & Gondhoraj & Rubiaceae & Shrub & $\mathrm{C}$ & Mar-May \\
\hline Ixora coccinia L., PS 111 & Rongon & Rubiaceae & Shrub & $\mathrm{C}$ & Jan-Dec \\
\hline $\begin{array}{l}\text { Neolamarckia cadamba (Roxb.) } \\
\text { Bosser, PS } 181\end{array}$ & Kadom & Rubiaceae & Tree & $\mathrm{C}$ & May-Jul \\
\hline
\end{tabular}


Table 1 Contd.

\begin{tabular}{|c|c|c|c|c|c|}
\hline $\begin{array}{l}\text { Scientific name and Voucher } \\
\text { number }\end{array}$ & Bangla name & Family & Habit & $\begin{array}{l}\text { Status of } \\
\text { occurrence }\end{array}$ & $\begin{array}{l}\text { Flowering } \\
\text { time }\end{array}$ \\
\hline Paederia foetida L., PS 250 & Gandhavaduli & Rubiaceae & Climber & $\mathrm{V}$ & Jan-Dec \\
\hline Ageratum conyzoides L., PS 112 & Ochunti & Asteraceae & Herb & $\mathrm{C}$ & Nov-Jun \\
\hline $\begin{array}{l}\text { Blumea lacera (Burm. f.) DC., PS } \\
182\end{array}$ & Borokucksim & Asteraceae & Herb & $\mathrm{C}$ & Nov-Jul \\
\hline $\begin{array}{l}\text { Chrysonthemum coronarium L., PS } \\
251\end{array}$ & $\begin{array}{l}\text { Chandro } \\
\text { mollika }\end{array}$ & Asteraceae & Herb & $\mathrm{C}$ & Dec-Feb \\
\hline $\begin{array}{l}\text { Chromolaena odorata (L.) King. \& } \\
\text { Robinson, PS } 330\end{array}$ & Germanlata & Asteraceae & Herb & $\mathrm{R}$ & Nov-May \\
\hline Cirsium arvense (L.) Scop., PS 113 & Shial Kanta & Asteraceae & Herb & $\mathrm{C}$ & Feb-Jun \\
\hline Cosmos bipinatus Cav., PS 183 & Cosmos & Asteraceae & Herb & $\mathrm{C}$ & Jan-Dec \\
\hline Eclipta alba (L.) Hassk, PS 252 & Kalokeshi & Asteraceae & Herb & $\mathrm{R}$ & Jan-Dec \\
\hline Enhydra fluctuans Lour., PS 114 & Helencha & Asteraceae & Herb & $\mathrm{C}$ & Jan-Apr \\
\hline Gnaphalium luteo-album L., PS 184 & Bara Kamra & Asteraceae & Herb & $\mathrm{C}$ & Mar-Aug \\
\hline $\begin{array}{l}\text { Grangea maderaspatana (L.) Poir., } \\
\text { PS } 253\end{array}$ & Namuti & Asteraceae & Herb & $\mathrm{R}$ & Dec-May \\
\hline Hellianthus annuus L., PS 115 & Surjomukhi & Asteraceae & Herb & $\mathrm{C}$ & Jan-Dec \\
\hline Lactuca sativa L., PS 185 & Lettuce & Asteraceae & Herb & $\mathrm{C}$ & Jan-Dec \\
\hline Launaea aspleniifolia DC., PS 254 & Tik-chana & Asteraceae & Herb & $\mathrm{C}$ & Jan-Aug \\
\hline $\begin{array}{l}\text { Mikania cordata (Burm f.) } \\
\text { Robinson, PS } 116\end{array}$ & Asamlata & Asteraceae & Climber & $\mathrm{C}$ & Oct-Feb \\
\hline Sonchus asper (L.) Hill., PS 186 & Sonpalong & Asteraceae & Herb & $\mathrm{C}$ & Sep-Jun \\
\hline Spilanthes acmella (L.)L., PS 255 & Marhatitiga & Asteraceae & Herb & $\mathrm{R}$ & Jan-Dec \\
\hline $\begin{array}{l}\text { Synedrella nodiflora (L.) Gaertn, PS } \\
304\end{array}$ & Relanodi & Asteraceae & Herb & $\mathrm{R}$ & Jan-Dec \\
\hline Tagetes erecta L., PS 117 & Genda & Asteraceae & Herb & $\mathrm{C}$ & Jan-Dec \\
\hline T. patula L., PS 187 & Gendaphul & Asteraceae & Herb & $\mathrm{C}$ & Nov-Mar \\
\hline Tridax procumbens L., PS 256 & Tridhara & Asteraceae & Herb & $\mathrm{C}$ & Jan-Dec \\
\hline Vernonia cinerea (L.) Less., PS 305 & Kuksim & Asteraceae & Herb & $\mathrm{C}$ & Jan-Dec \\
\hline $\begin{array}{l}\text { Wedelia trilobata (L.) A.S. Hitchc., } \\
\text { PS } 331\end{array}$ & Keshraj & Asteraceae & Herb & $\mathrm{C}$ & Jan-Dec \\
\hline $\begin{array}{l}\text { Xanthium indicum Koen ex Roxb., } \\
\text { PS } 257\end{array}$ & Ghagra & Asteraceae & Herb & $\mathrm{C}$ & Jan-Dec \\
\hline Youngia japonica (L.) DC., PS 118 & Youngful & Asteraceae & Herb & $\mathrm{R}$ & Aug-Jan \\
\hline $\begin{array}{l}\text { Zinnia pauciflora L., PS } 188 \\
\text { LILIOPSIDA }\end{array}$ & Zinnia & Asteraceae & Herb & $\mathrm{R}$ & Jun-Aug \\
\hline Areca catechu L., PS 119 & Shupari & Arecaceae & Tree & $\mathrm{C}$ & Jan-Dec \\
\hline Borassus flabellifer L., PS 189 & Taal & Arecaceae & Tree & $\mathrm{C}$ & Jan-Oct \\
\hline Cocos nucifera L., PS 258 & Narkel & Arecaceae & Tree & $\mathrm{C}$ & Jan-Dec \\
\hline Phoenix sylvestris Roxb., PS 120 & Khejur & Arecaceae & Tree & $\mathrm{C}$ & Dec-May \\
\hline $\begin{array}{l}\text { Alocasia macrorrhizos (L.) G. Don., } \\
\text { PS } 190\end{array}$ & Mankochu & Araceae & Herb & $\mathrm{C}$ & Jul-Oct \\
\hline $\begin{array}{l}\text { Amorphophallus campanulatus } \\
\text { (Roxb) BI. ex. Dense, PS } 121\end{array}$ & Olkochu & Araceae & Herb & $\mathrm{C}$ & May-Nov \\
\hline $\begin{array}{l}\text { Colocasia esculenta (L.) Schott., PS } \\
191\end{array}$ & Kochu & Araceae & Herb & $\mathrm{C}$ & May-Oct \\
\hline
\end{tabular}


Table 1 Contd.

\begin{tabular}{|c|c|c|c|c|c|}
\hline $\begin{array}{l}\text { Scientific name and Voucher } \\
\text { number }\end{array}$ & Bangla name & Family & Habit & $\begin{array}{c}\text { Status of } \\
\text { occurrence }\end{array}$ & $\begin{array}{l}\text { Flowering } \\
\text { time }\end{array}$ \\
\hline $\begin{array}{l}\text { Epipremnum pinnatum (L.) Engl., } \\
\text { PS PS } 259\end{array}$ & Money plant & Araceae & Climber & $\mathrm{R}$ & Apr-May \\
\hline $\begin{array}{l}\text { Typhonium trilobatum (L.) Schott, } \\
\text { PS } 306\end{array}$ & Camgash & Araceae & Herb & $\mathrm{C}$ & Apr-Oct \\
\hline Lemna perpusilla Torrey, PS 122 & Khudipana & Lemnaceae & Herb & $\mathrm{C}$ & Jan-Dec \\
\hline Commelina benghalensis L., PS 192 & Kanshira & Commelinaceae & Herb & $\mathrm{C}$ & Apr-Nov \\
\hline Cyanotis cristata Schutt., PS 260 & Kendara & Commelinaceae & Herb & $\mathrm{C}$ & Sep-Feb \\
\hline Cyperus rotundus L., PS 123 & Muthagas & Cyperaceae & Herb & $\mathrm{C}$ & Sep-Feb \\
\hline $\begin{array}{l}\text { Kyllinga nemoralis (J.R. Forst. \& } \\
\text { G. Forst.) Dandy ex Hutchins. \& } \\
\text { Dal., PS } 124\end{array}$ & Nirbishi & Cyperaceae & Herb & $\mathrm{C}$ & Jun-Sep \\
\hline $\begin{array}{l}\text { Bambusa bambos (L.) Voss., PS } \\
193\end{array}$ & Bash & Poaceae & Shrub & $\mathrm{C}$ & Jan-Dec \\
\hline Cynodon dactylon (L) Pers., PS 261 & Durba & Poaceae & Herb & $\mathrm{C}$ & Jan-Dec \\
\hline Oryza sativa L., PS 125 & Dhan & Poaceae & Herb & $\mathrm{C}$ & Jul-Sep \\
\hline Saccharum officinarum L., PS 194 & Aakh & Poaceae & Shrub & $\mathrm{C}$ & Jan-Dec \\
\hline Setaria glauca (L.) Beauv, PS 262 & Kawn & Poaceae & Herb & $\mathrm{C}$ & Jan-Dec \\
\hline Triticum aestivum L., PS 307 & Gom & Poaceae & Herb & $\mathrm{C}$ & Jan-Dec \\
\hline Zea mays L., PS 126 & Vutta & Poaceae & Shrub & $\mathrm{C}$ & Mar-Apr \\
\hline Ananas comosus (L.) Merr., PS 195 & Anaras & Bromeliaceae & Herb & $\mathrm{R}$ & Feb-Jul \\
\hline Musa paradisiaca L., PS 263 & Kola & Musaceae & Herb & $\mathrm{C}$ & Jan-Dec \\
\hline Cucurma longa L., PS 127 & Holud & Zingiberaceae & Herb & $\mathrm{C}$ & Mar-Oct \\
\hline Zingiber officinale Rose, PS 196 & Ada & Zingiberaceae & Herb & $\mathrm{C}$ & Mar-Aug \\
\hline $\begin{array}{l}\text { Cheilocostus speciosus (J.Koenig) } \\
\text { C. Specht., PS } 264\end{array}$ & Keumul & Costaceae & Herb & $\mathrm{R}$ & Sep-Dec \\
\hline Canna indica L., PS 128 & Kolaboti & Cannaceae & Herb & $\mathrm{C}$ & Apr-Nov \\
\hline Allium cepa L., PS 197 & Piyaj & Liliaceae & Herb & $\mathrm{C}$ & Feb-Jun \\
\hline A. sativum L., PS 265 & Rosun & Liliaceae & Herb & $\mathrm{C}$ & Feb-Apr \\
\hline $\begin{array}{l}\text { Asparagus racemosus Willd., PS } \\
308\end{array}$ & Satamili & Liliaceae & Climber & $\mathrm{R}$ & Nov-Mar \\
\hline Aloe vera (L.) Burn.f., PS 129 & Ghrita kumari & Aloeaceae & Herb & $\mathrm{C}$ & Sep-Dec \\
\hline Smilax macrophylla Roxb., PS 198 & Kumarilata & Smilaceae & Climber & $\mathrm{R}$ & Nov-Mar \\
\hline Dioscorea alata L., PS 130 & Chupri alu & Dioscoriaceae & Climber & $\mathrm{R}$ & Oct-Dec \\
\hline $\begin{array}{l}\text { Vanda tessellata (Roxb.) Hook.f., } \\
\text { PS } 199\end{array}$ & Rasna & Orchidaceae & $\begin{array}{l}\text { Epiphyti } \\
\text { c Herb }\end{array}$ & $\mathrm{R}$ & Apr-Jun \\
\hline
\end{tabular}

Jan = January, Feb $=$ February, Mar $=$ March, Apr $=$ April, May $=$ May, Jun = June, Jul = July, Aug = August, Sep $=$ September, Oct $=$ October, Nov $=$ November, Dec $=$ December, $\mathrm{C}=$ Common, $\mathrm{R}=$ Rare, Vul $=$ Vulnerable, $\mathrm{E}=$ Endangered.

genera 108 families were recorded in Rajshahi district (Rahman, 2013). A total of 302 species belonging to 243 genera under 84 families were recorded in Bangladesh Police Academy, Rajshahi (Rahman et. al, 2014). But there has been published information on the diversity of angiosperm plant species in Gobindaganj of Gaibandha district, Bangladesh.

The study area has a moderately rich resource of angiosperms, it witness some threats which might drive this resource to an endangered stated. Observations and group discussion with local people during field works resulted in identifying some major threats which include urbanization, 
modern agriculture, brick fields, deforestation, and lack of awareness, exotic plantation and river erosion. Therefore, efforts should be undertaken to safeguard the plants through ex situ and in situ conservation approaches, public awareness, and ensuring protection of habitats.

\section{Acknowledgements}

The authors are grateful to the Ministry of Science and Technology (MOST), Government of the People's Republic of Bangladesh for financial support to complete this research work. The authors are also thankful to the local people in Gobindaganj upazila of Gibandha district, Bangladesh for their co-operation and help during the research work.

\section{References}

Ahmed, Z.U., Begum, Z.N.T., Hassan, M.A., Khondker, M., Kabir, S.M.H., Ahmad, M., Ahmed, A.T.A., Rahman, A.K.A. and Haque, E.U.(Eds). 2008-2009. Encyclopedia of Flora and Fauna of Bangladesh. Vols. 6-10. Asiatic Society of Bangladesh, Dhaka.

Arefin, M.K., Rahman, M.M., Uddin, M.Z. and Hassan, M.A. 2011. Angiosperm Flora of Satchari National Park, Habiganj, Bangladesh. Bangladesh J. Plant Taxon. 18(2): 117-140.

Bangladesh Population Census (BPC) 2001. Bangladesh Bureau of Statistics; Cultural survey report of Gobindhaganj Upazila 2007.

Christenhusz, M.J.M and Byng, J.W. 2016. The number of known plants species in the world and its annual increase. Phytotaxa, 261(3): 201-217.

Hooker, J. D. 1877 (rep. ed. 1961).Flora of British India.Vols.1-7. L. Reeve and Co. Ltd. London, U.K.

Huq, A.M. 1986. Plant Names of Bangladesh.Bangladesh National Herbarium, BARC, Dhaka, Bangladesh.

Islam, M.R., Uddin,M.Z. and Hassan, M.A. 2009. An Assessment of the Angiosperm Flora of Ramgarh Upazila of Khagrachhari District, Bangladesh. Bangladesh J. Plant Taxon. 16(2): 115-140.

Khan, M.S. and Afza, S.K. 1968.A taxonomic report on the angiospermic flora of Teknaf and St. Martin's Island. Dhaka Univ. Studies, Part B. 16: 35-37.

Khan, M.S. and Banu, F. 1972. A taxonomic report on the angiospermic flora of Chittagong Hill Tracts- 2. J. Asiat. Soc. Bangladesh. 17(2): 63-68.

Khan, M.S. and Hassan, M.A. 1984. A taxonomic report on the angiospermic flora of St.Martin's Island. Dhaka Univ. Studies, Part B. 32(1): 76-78.

Khan, M.S. and Huq, A.M. 2001.The vascular flora of Chunati Wildlife Sanctuary in south Chittagong, Bangladesh. Bangladesh J. Plant Taxon. 8(1): 47-64.

Lindley, J. 1830. Introduction to the Natural System of Botany.Longman. London, UK.

Moniruzzaman, M., Hassan, M. A., Rahman, M.M., Layla, S. and Islam, M.R. 2012. A Preliminary Checklist of the Angiospermic Flora of DaulotpurUpazila in Kushtia District, Bangladesh. J. Asiat. Soc. Bangladesh, Sci. 38(1): 53-65.

Naik, V.N. 2003.Taxonomy of Angiosperms. Tata McGraw-Hill Publishing Company Limited, New Delhi. India.

Pasha, M. K. and Uddin, S. B. 2013. Dictionary of Plant Names of Bangladesh (Vascular Plants). Janokalyan Prokashani. Chittagong, Dhaka, Bangladesh.

Prain, D. 1903 (rep. ed. 1963).Bengal Plants.Vols.1-2. Botanical Survey of India. Calcutta, India.

Rahman, A.H.M.M. 2013. Angiospermic flora of Rajshahi district, Bangladesh. American Journal of Life Sciences.1(3): 105-112.

Rahman, A.H.M.M., Ferdous, Z. and Islam, A. K. M. R. 2014. A Preliminary Assessment of Angiosperm Flora of Bangladesh Police Academy. Research in Plant Sciences. 2(1): 9-15.

Rahman, M.O. and Alam, M.T. 2013. A taxonomic study on the angiosperm flora of Trishal Upazilla, Mymensingh. Dhaka Univ. J. Biol. Sci. 22(1): 63-74. 
Rahman, M.O., Begum, M. and Ullah, M.W. 2013. Angiosperm Flora of Sadar Upazila of Munshiganj District, Bangladesh. Bangladesh J. Plant Taxon. 20(2): 213-231.

Rahman, M.O. and Hassan, M.A. 1995. Angiospermic flora of Bhawal National Park, Gazipur (Bangladesh). Bangladesh J. Plant Taxon. 2(1\&2): 47-79.

Tutul, E., Uddin, M.Z., Rahman, M.O. and Hassan, M.A. 2010. Angiospermic Flora of Runctia Sal Forest, Bangladesh. II. Magnoliopsida (Dicots). Bangladesh J. Plant Taxon. 17(1): 33-54.

Uddin, M.Z., Alam, M.F., Rahman, M.A. and Hassan, M.A. 2013. Diversity in Angiosperm Flora of Teknaf Wildlife Sanctuary, Bangladesh. Bangladesh J. Plant Taxon. 20(2): 145-162.

Uddin, M.Z. and Hassan, M.A. 2010. Angiosperm Diversity of Lawachara National Park (Bangladesh): A Preliminary Assessment. Bangladesh J. Plant Taxon. 17(1): 9-22.

Uddin, S.N. and Hassan, M.A. 2012. Angiosperm Flora of Rampahar Reserve Forest under Rangamati District in Bangladesh. I. Liliopsida (Monocots). Bangladesh J. Plant Taxon. 19(1): 37-44.

(Manuscript received on 4 June, 2019; revised on 4 December, 2019) 